\title{
PROTEOMIC ANALYSIS
}

\section{New tool for proteomic analysis}

Shen, X. et al. PNAS 115, E4767-E4776 (2018).

As proteins are the molecular machinery of the cell, proteomics is a natural choice to characterize biochemical processes. The preferred analytical method for proteomics is mass spectrometry (MS) because of its sensitivity and ability to characterize unknown molecules. Since intact proteins are difficult to examine via MS, most studies digest proteins into peptides. However, digests of tissue extracts are complex, containing hundreds of thousands of peptides. A mass spectrometer cannot process all of these peptides simultaneously, so digests are usually separated using liquid chromatography (LC) prior to analysis. As no two LC runs are the same, matching peptides from different runs is challenging and lining up all of the data in large cohort studies is extremely difficult. Additional problems with LC-MS include drift in the instrument's sensitivity and chemical noise that can complicate measurement of peptides.
In a new report, the laboratory of Jun $\mathrm{Qu}$, a professor in Pharmaceutical Science from the University at Buffalo, present a software package, IonStar, that addresses these difficulties with proteomic analysis. To begin, the team benchmarked IonStar with a control sample and compared it to other analysis packages. Their software quantified more proteins and had the least missing data of all the programs while also having the lowest false discovery rate. Finally, IonStar had the best precision and accuracy.

Next, investigators applied the software to a real dataset that looked at traumatic brain injury (TBI). Each year millions of people suffer TBI-classified as mild, moderate, or severe-and the search for therapeutic drugs is a priority at the National Institute of Neurological Disorders and Stroke. Another professor from the University at Buffalo, David Poulsen, had characterized two drugs, methamphetamine (METH) and phenoxybenzamine (PBZ), that reduced damage following TBI in rats as assayed through cognitive and behavioral tests.

The experiment consisted of 5 groups of 10 rats subjected to mild TBI, extreme TBI, extreme TBI with PBZ treatment 8 hours post injury, extreme TBI with METH treatment 8 hours post injury, and sham animals. At 32 hours, the researchers collected tissues from hippocampus and cortex. Upon analysis, they quantified 7200 proteins, with only $0.2 \%$ of the proteins having missing values. Proteomic results resembled neurobehavioral work in that the two drug treatments made severe TBI animals resemble mild TBI.

Future plans for IonStar include improving identification of unassigned peptides to increase protein coverage.

\section{Clark Nelson}

Published online: 24 July 2018

https://doi.org/10.1038/s41684-018-0127-3

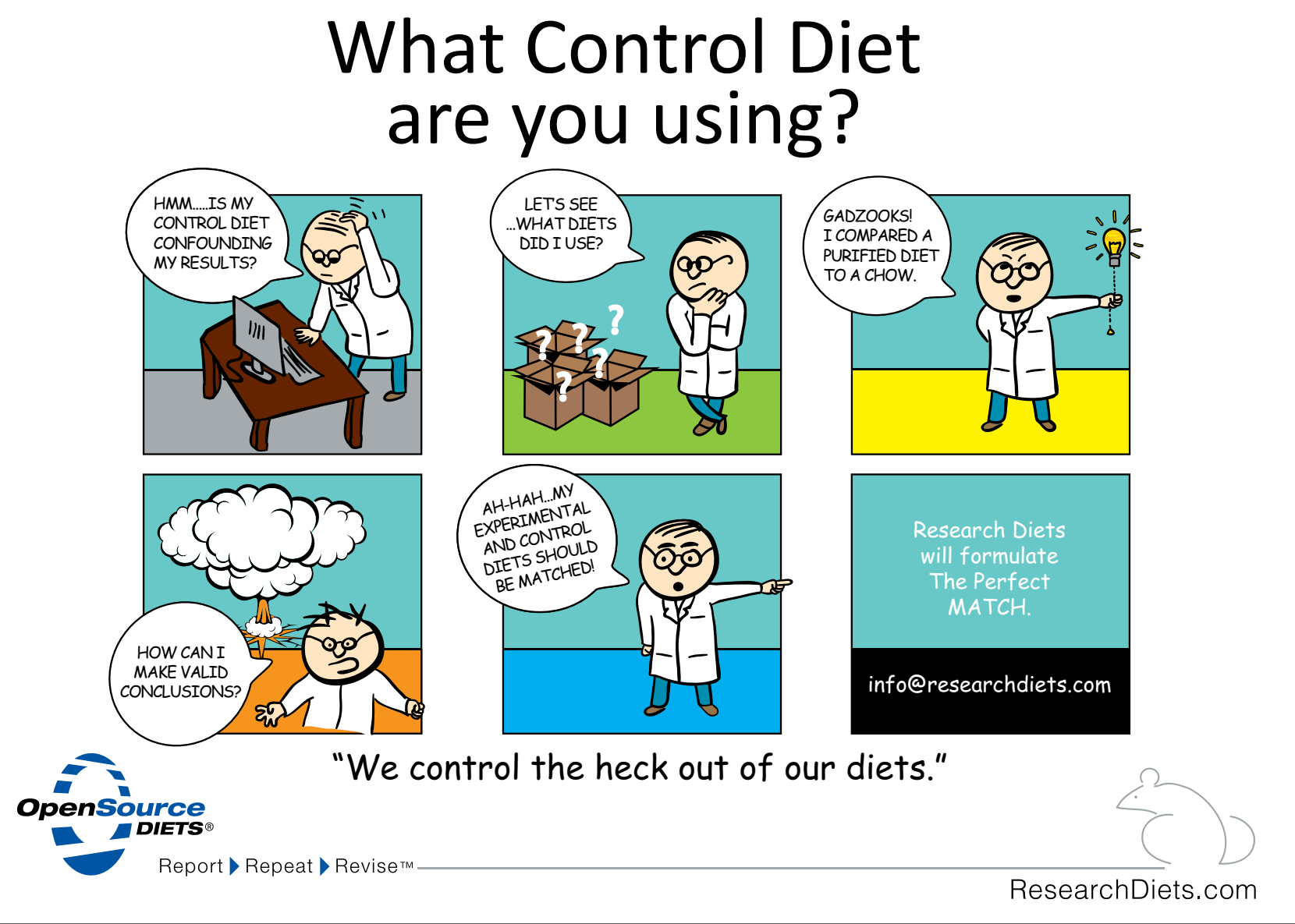

